\title{
The therapeutic features of acute kidney injury in the elderly
}

\author{
Blebea Nicoleta Mirela $^{1 *}$, Stăniguț Alina ${ }^{2}$, Mitea Gabriela ${ }^{1}$ \\ ${ }^{1}$ Ovidius University of Constanta, Faculty of Pharmacy, Constanta, Romania, \\ ${ }^{2}$ Internal Medicine- Nephrology Department, Emergency Clinical County Hospital, Faculty of \\ Medicine, Ovidius University of Constanta, Romania, \\ *Corresponding author: Nicoleta Mirela Blebea, nicoleta.blebea@gmail.com
}

\begin{abstract}
The incidence of acute kidney damage (AKI) is increasing in people of all ages, but patients over the age of 65 are more likely to develop AKI due to structural and functional renal failure associated with aging, comorbidities and reduced ability to recover. Older patients with AKI have an increased risk of mortality in both the short and long term, and survivors are patients with chronic kidney disease (CKD) who eventually progress to end-stage renal disease (ESRD). This paper aims at the therapeutic management of the elderly suffering from AKI. It is also intended to show the therapeutic methods taken for these patients to which are added nonpharmacological measures, such as maintaining adequate hydration and avoiding nephrotoxic agents, which are helpful in preventing AKI in the elderly.
\end{abstract}

Keywords: acute kidney injury, acute renal failure, elderly.

\section{Introduction}

AKI is the rapid loss of renal function, which occurs in hours or days and results in the accumulation of metabolic wastes and affect the extracellular volume and electrolyte homeostasis. AKI is common, especially in the elderly, over the age of 65 . Studies show that about 2-7\% of all hospitalizations elderly patients are affected by AKI. Acute kidney disease has been consistently associated with increased morbidity and mortality, and multiple studies, as well as a meta-analysis, have shown poorer results in the elderly [1], [2]. Annually, AKI affects approximately $0.3-0.5 \%$ of adults and $25-70 \%$ of critically ill patients. Aging is associated with decreased organ function and the occurrence of chronic diseases. An analysis in the U.S. showed that the AKI rate gradually increases in patients over 65 years of age. This trend is valid for both AKI requiring dialysis and AKI requiring dialysis. However, many studies specifically address AKI in the elderly population, with a special focus on the inherent differences between the elderly and the general population [3], [4]. Moreover, elderly patients often have comorbidities, including diabetes, hypertension, oncological disease, infections and cardiovascular disease, and are under many different medications, making pharmacological assessment complex in these patients and leading to decreased estimated glomerular filtration rate (GFR), hypoalbuminemia, or retention of "uremic" dissolved substances, any of these is poor physical performance and fragility [5], [6]. Definition and staging of AKI are based on the Risk, Injury, Failure; Loss, End-Stage Kidney 
Disease (RIFLE), classification introduced in 2004, allowing the description of the presence of acute renal damage (AKI) and the definition of its clinical stage, based on serum creatinine ( $\mathrm{SCr}$ ) and urine flow (OU). The SCr and UO criteria have also been used to develop other scoring systems, such as the Acute Renal Injury Network (AKIN) and the Renal Disease Improving Global Outcome (KDIGO). Current practice suggests that the RIFLE, AKIN diagnostic criteria and KDIGO used to assess the presence and severity of AKI are insufficient to illustrate the complexity of AKI syndrome [7]. Numerous renal anatomical and functional changes occur in elderly patients, leading to a reduced GFR, decreased renal blood flow and impaired renal self-regulation [8].

\section{Causes of AKI}

The elderly is particularly vulnerable to the development of renal dysfunction and AKI is a common problem [1]. Common iatrogenic combinations include pre-existing renal dysfunction and exposure to nephrotoxic substances (carbon tetrachloride, ethylene glycol) or nephrotoxic drugs (aminoglycosides, contrast substances, NSAIDs) [9], [10], [11], and the use of ACE inhibitors and diuretics in patients with atherosclerosis. The etiology of AKI is grouped into three categories: prerenal, renal and postrenal [8], [9]. Prerenal AKI is the second most common cause of AKI in the elderly, accounting for nearly onethird of all hospitalized cases. insufficient irrigation of the kidneys (dehydration, haemorrhage, burns, diarrhea or intense vomiting, anaphylactic, septic or other shock, blocking the transit of blood on the renal vessels by embolism or thrombosis) [1], [9]. Delay with the treatment of prerenal IRAs can lead to the development of renal IRAs, respectively kidney damage. The most frequent causes of AKI that were of medical etiology were: fluid loss due to gastrointestinal reasons nephrotoxic drugs, sepsis, other nephrotoxic substances [11]. Acute tubular necrosis (ATN) is the most common cause of intrinsic AKI and is responsible for over $50 \%$ of AKI in hospitalized patients and up to $76 \%$ of cases in patients from intensive care units. The probability of a postrenal cause for AKI increases with age. Benign prostatic hypertrophy, prostate carcinoma and pelvic malignancies, or external compressions caused by tumors, retroperitoneal fribrosis are all important causes [1], [8]. Early identification of AKI secondary to obstruction with imagistic investigations is essential, and complete or partial renal recovery usually results after removing the obstruction [9], [10], [11].

\section{Clinical Manifestations}

The symptoms of AKI range from almost absent clinical manifestations to very severe conditions and are strongly influenced by the patient's previous condition and the concrete cause of the AKI. In most cases, the sign is oliguria or anuria. It can be associated with general weakness, general malaise, fatigue, confusion, nausea, vomiting, muscle cramps, metallic taste in the mouth. In severe cases, the onset of AKI can be with a coma or seizures [9], [10], [11].

\section{Diagnosis}

The diagnosis is established by anamnesis, physical examination and laboratory tests (creatinine, urea, Clearance of creatinine, Urinary flow is decreased and urine analysis often shows the presence of leukocytes, erythrocytes and proteins. In order to specify the cause of AKI and differentiate from CKD, additional imaging investigations such as ultrasonography, computed tomography may be required [8], [9], [10], [11] .

The diagnosis of AKI must be established early, from the first hours after the patient's presentation in a medical service, with early applied therapeutic measures that increase the chances of rapid recovery of renal function. Another diagnosis problem is related to establishing the etiology of AKI because the 
therapeutic approach is different. Rigorous history and complete clinical and paraclinical examination are key elements for early diagnosis.

\section{Treatment}

The treatment of ARF varies depending on the form and severity of the pathology. Therapeutic measures can be administered concomitantly or successively. Treatment include: general measures, etiological treatment, treatment of complications, pathogenic treatment.

It is recommended to limit the intake of salt and protein; fluid intake is adjusted to diuresis and extrarenal loss. Stop administration of nephrotoxic drugs is required. The treatment of associated renal and extrarenal pathologies is instituted. The main treatment methods include: maintaining fluid and electrolyte balance in case of excessive renal / extrarenal loss or secondary to generalized vascular dilatation (prerenal AKI), surgical correction of urinary tract obstruction or compression (postrenal AKI), administration of antidotes in certain cases of intoxication. In severe AKI, hemodialysis or peritoneal dialysis is performed [11], [12], [13], [14], [15].

\subsection{Non-drug treatment}

\subsubsection{Diet recommended for AKI patients}

Water intake during oligo-anuria, daily fluid intake, at each additional $5^{\circ} \mathrm{C}$ of the environment $\left(\geq 25^{\circ} \mathrm{C}\right)$ and at each additional $1^{\circ} \mathrm{C}$ of body temperature $\left(\geq 37^{\circ} \mathrm{C}\right)$, it is recommended to increase the water intake by 0.5-1 L / day. In hypertensive and oedematous patients, the water intake decreases by $0.5-1 \mathrm{~L} /$ day, compared to the recommended one until the normalization of blood pressure values and the disappearance of oedema [16], [17].

During the polyuria phase: fluid intake is calculated according to the same formula, it is important to ensure adequate fluid intake, thus avoiding a large number of complications. Daily weighing is important in assessing water balance [16], [17].

During the phase of oligoanuria and polyuria, it is recommended to administer thermally processed products, easily digestible, without the addition of spices. Excludes fatty varieties of meat (goose, duck, pork, goat, lamb) and fish, sour, fried snacks, salty and smoked foods, bacon, legumes, coffee, tea and hard cocoa, alcoholic beverages [18].

\section{Caloric intake}

Mild / moderate AKI: 30-50 kkal / kg / day; severe, hypercatabolic AKI: up to 60-70 kkal / kg / day. When limiting oral administration, it is recommended to initiate parenteral nutrition with the recommended caloric intake, amino acid solutions [18].

\section{Protein intake}

During the oligo-anuric phase proteins are limited to $0.6-0.8 \mathrm{~g} / \mathrm{kg} /$ day. Preferably are animal or soy proteins. Parenteral administration of amino acid solutions of $200-400 \mathrm{ml} /$ day is recommended. During the polyuria / recovery phase about $1 \mathrm{~g} / \mathrm{kg} /$ day of protein.

The intake of lipids should be $0.7-1.0 \mathrm{~g} / \mathrm{kg} / \mathrm{day}$, at least $1 / 3$ of the fats must be of vegetable origin (polyunsaturated fatty acids).

In the phase of oligo-anuria, easily digestible carbohydrates $4-5 \mathrm{~g} / \mathrm{kg} / \mathrm{day}$ are preferred, and potassium is limited and in the phase of polyuria it is often necessary its additional administration (fruits, juices, compotes, etc. or in intravenous or oral solution).

Phosphorus, it is limited in the period of oligo-anuria (meat, fish, dairy products) [18]. 


\subsubsection{Dietary supplements recommended for AKI patients}

Vitamins and antioxidants, it is recommended tocopherol acetate 400 U.I, 1-2 capsules / day, ascorbic acid compressed $0.5 \times 3$ times / day, thiamine, riboflavin, pyridoxine and cyanocobalamin - initially parenteral in regular daily doses, after stabilizing the patient's condition [18].

It is recommended to limit and avoid emotional stress and graduate increased physical exertion.

The principles of drug treatment in the elderly include the treatment of basic pathology depending on the evolution phase and etiopathogenetic form, drug strategies to prevent aggravation of renal damage in AKI, emergency drug treatment, etiopathogenetic drug treatment, symptomatic treatment of AKI complications, nephroprotective treatment that prevent the transformation of the AKI in to CKD [11], [12], [13], [14], [15].

\subsection{Therapeutic treatment}

In hypotension it is recommended treatment with norepinephrine and or high doses (10-15 drops / min) of dopamine.

In hypovolemic patient it is recommended treatment with colloidal solutions (albumin), crystalline solutions (sodium chloride), dextran.

In oliguria and tubular obstruction, it is recommended treatment with loop diuretics (furosemide), osmotic diuretics (mannitol).

In renal causes of AKI, it is recommended treatment with vasoconstriction low doses (5-7 drops / min) of dopamine, calcium channel blockers (BCC) (nifedipine, verapamil), PAF antagonists and leukotrienes [12], [13], [14], [15].

\subsubsection{Emergency management}

In hyperkalemia it is recommended treatment with $40 \%$ glucose - $20 \mathrm{ml}$; insulin (fast-acting) $2.4 \%$ sodium bicarbonate $-200 \mathrm{~mL}$ the last one depending on the presence of acidosis; $\beta$-adrenomimetics (salbutamol). The control is performed according to the level of potassium in the blood $(\leq 6.5 \mathrm{mmol} /$ $\mathrm{L}$, especially $\leq 5.5 \mathrm{mmol} / \mathrm{L}$ ) and the disappearance of the signs of hyperkalaemia on the ECG.

In pulmonary oedema it is recommended treatment with loop diuretics (furosemide, bumetanide); peripheral vasodilators (nitrates).

In cerebral oedema it is recommended treatment with osmotic diuretics (mannitol), magnesium sulfate.

In local gastrointestinal bleeding it is recommended treatment with antisecretory drugs (famotidine, omeprazole) [19], somatostatin-in bleeding due to rupture of gastroesophageal varices in liver cirrhosis, volume support (colloidal solutions, crystalloids, erythrocyte mass), surgical treatment (open or endoscopic surgery).

In hypertensive crisis it is recommended treatment with antihypertensive (loop diuretic-furosemide, bumetanide), calcium channel blockers - CCB (diltiazem, verapamil, nifedipine, amlodipine) / $\beta$-blocker (such as nebivolol) and central drugs.

In shock it is recommended to administer volume support (crystalline and colloidal solutions), vasoconstrictor drugs (norepinephrine, phenylephrine) [20].

In venous thrombosis it is recommended to administer antiplatelet, direct anticoagulants (heparin and low molecular weight heparins) and indirect (warfarin, acenocumarol).

In pulmonary embolism it is recommended to administer thrombolytics (streptokinase), antiplatelet drugs (aspirin - acetylsalicylic acid, clopidogrel), direct anticoagulants (heparin and low molecular weight heparins) and indirect (warfarin, acenocumarol), oxygen therapy [11], [12], [13], [14], [15]. 


\subsubsection{Symptomatic treatment of AKI complications}

In metabolic acidosis it is recommended limitation of protein intake ( $<0.8 / \mathrm{kg} \mathrm{x}$ day), administration of sodium bicarbonate with achievement of blood bicarbonate values $>22 \mathrm{mmol} / \mathrm{L}$ and $\mathrm{pH}>7.35$.

In hyperphosphatemia it is recommended limitation of phosphate intake (meat, fish, dairy products) $<800 \mathrm{mg} /$ day [18], intestinal phosphate chelators - calcium acetate, calcium carbonate, aluminium hydroxide.

In hypocalcaemia it is recommended to administer of sol. calcium gluconate $10 \%-10-20 \mathrm{~mL}$ sol. sodium bicarbonate.

In hypermagnesemia it is recommended discontinuation of magnesium supplements (if administered).

In hyperuricemia is necessary a special treatment and is required in cases where uric acid is $>7.5 \mathrm{mg}$ / $\mathrm{dL}$ or $>900 \mu \mathrm{mol} / \mathrm{L}$ and is not increased in the context of dehydration [11], [12], [13], [14], [15].

\section{Conclusions}

The clinical characteristics for AKI are variable. The therapeutic approach for patients presenting AKI it is recommended to be supportive. All elderly patients with AKI require close monitoring of electrolyte and acido-basic imbalances and nutrition. Rapid and effective correction of renal perfusion deficit determines the return to normal renal function and avoids the transition to organic IRA (severe and prolonged renal hypoperfusion leads to acute ischemic tubular necrosis). Clinical and biological improvement allows the etiological treatment. Nutritional support should not be overlooked, as nutritional status is an important predictor of prognosis in AKI, and the elderly are at particular risk of developing malnutrition.

\section{References}

[1] K. ABDEL-KADER, P. M. PALEVSKY: Acute kidney injury in the elderly. Clin Geriatr Med., 25 (3), 331-358 (2009).

[2] K. MAKRIS, L. SPANOU: Acute Kidney Injury: Definition, Pathophysiology and Clinical Phenotypes. Clin Biochem Rev., 37(2), 85-98, (2016).

[3] C. CHIA -TER, T. HUNG-BIN, L. YU-FENG, K. WEN-JE: Acute kidney injury in the elderly: Only the tip of the iceberg. Journal of Clinical Gerontology and Geriatrics, 5(1), 7-12, (2014).

[4] J. L. HARDING, Y. LI , N. R. BURROWS NR, K. M. BULLARD, M. E. PAVKOV: US Trends in Hospitalizations for Dialysis-Requiring Acute Kidney Injury in People With Versus Without Diabetes. Am J Kidney Dis. June 2020, 75(6), 897-907.

[5] C. PONTICELLI, G. SALA G, R.J. GLASSOCK: Drug management in the elderly adult with chronic kidney disease: a review for the primary care physician. Mayo Clin Proc. 2015,90 (5), 633-645.

[6] J. R. PETRIE, T. J. GUZIK, R. M. TOUYZ: Diabetes, Hypertension, and Cardiovascular Disease: Clinical Insights and Vascular Mechanisms. Can J Cardiol. 2018, 34(5), 575-584.

[7] J. A. LOPES, S. JORGE: The RIFLE and AKIN classifications for acute kidney injury: a critical and comprehensive review. Clinical Kidney Journal, 2013, 6(1), 8-14.

[8] A. FARRAR: Acute Kidney Injury. The Nursing clinics of North America . 2018, 53(4), 499-510.

[9] C. RONCO, L. M. RIZO-TOPETE, M. SERRANO SOTO: Acute Kidney Injury: From Clinical to Molecular Diagnosis, Critical Care Nephrology (Third Edition), Elsevier, Chapter 11, 2019 , 65-72. 
[10] C. RONCO, L. RIZO-TOPETE, M. SERRANO-SOTO, K. KASHANI: Pro: Prevention of acute kidney injury: time for teamwork and new biomarkers. Nephrology Dialysis Transplantation, 2017, 32 ( 3), 408-413.

[11] L. TUTA, A. STANIGUT,, B. CAMPINEANU: Medical causes of acute kidney injury-clinical and therapeutic aspects in Constanta county. ARS Medica Tomitana, 2012, 2(69), 66-71.

[12] R. BELLOMO, C. RONCO, J. KELLUM, R. L. MEHTA, P. PALEVSKY: Acute renal failure definition, outcome measures, animal models, fluid therapy and information technology needs: the second international consensus conference of the Acute Dialysis Quality Initiative Group (ADQI). Crit. Care. 2004, 8 (4), 204-212.

[13] A. COVIC, M. COVIC, L. SEGALL, P. GUSBETH-TATOMIR: Handbook of Nephrology, chapter: Acute renal failure, Polirom Publishing House, Bios, Iași, 2007, 260.

[14] S. LEVEY: Humes D. Chapter 17 "Acute renal failure," in Malluche H. et al. Clinical Nephrology, Dialysis and Transplantation, Lippincott, 2007, 569-605.

[15] N. KIERAN, H. BRADY, A. JEFFERSON, R. ZAGER, I. CHENG, R. : Acute Renal Failure. Comprehensive Clinical Nephrology, 2nd edition, edited by R.J. JOHNSON and J. FEEHALLY, Mosby, Edinburgh - London - New York, 2003, 183-242.

[16] W. F. ClARK, S. H. HUAN, A. X. GARG, K. Gallo, A. A. House, L. Moist, M. A. Weir, J. M. Sontrop: The Chronic Kidney Disease Water Intake Trial: Protocol of a Randomized Controlled Trial. Can J Kidney Health Dis., 2017, 4.

[17] S. STICKEL, W. GIN-SING, M. WAGENAAR, J. S. R. GINNS : The practical management of fluid retention in adults with right heart failure due to pulmonary arterial hypertension. Eur Heart J Suppl. 2019, 21, 46-53.

[18] A. GOSTNSKA, M. STAWNY, K. DETTLAFF, A. JELINSKA: Clinical Nutrition of Critically Ill Patients in the Context of the Latest ESPEN Guidelines. Medicina (Kaunas). 2019, 55(12), 770.

[19] L. A. TUTA, A. STANIGUT, C. PANA, B. CAMPINEANU: The impact of proton pumps inhibitors withdrawal in patients with chronic kidney disease. Nephrology Dialysis Transplantation. 2019, 34(1).

[20] C. PANA , A. M. STANIGUT: Acute Kidney injury in sepsis in the eldery. 5th International Multidisciplinary Scientific Conference on Social Sciences and Arts SGEM 2018, 13-18. 